\title{
Granular cell tumors of the breast: an unusual case of multifocality-a case report
}

\author{
Sravya Chilukuri ${ }^{1}$, Raghavendra Pillappa ${ }^{2}$, Kelley Allison $^{3}$, Harry D. Bear ${ }^{1}$ \\ ${ }^{1}$ Division of Surgical Oncology, Departments of Surgery and Microbiology and Immunology and the Massey Cancer Center, Virginia \\ Commonwealth University, Richmond, VA, USA; ${ }^{2}$ Division of Anatomic Pathology, Department of Pathology, Virginia Commonwealth University \\ Health System, Richmond, VA, USA; ${ }^{3}$ Division of Breast Imaging, Department of Diagnostic Radiology, Virginia Commonwealth University Health \\ System, Richmond, VA, USA \\ Correspondence to: Harry D. Bear, MD, PhD. Division of Surgical Oncology, 1200 East Broad Street, 4th Floor South Wing, Box 980011 Richmond, \\ VA 23298-0011, USA. Email: harry.bear@vcuhealth.org.
}

\begin{abstract}
A 39-year-old female with no prior cancer diagnosis presented with a right breast lump that increased in pain with menstruation over the past 4 years. Imaging demonstrated two masses in the right breast and one mass in the left breast and image-guided core needle biopsy was consistent with bilateral granular cell tumors (GCTs) of the breasts. Based on the multifocal nature of the patient's breast masses and associated pain, diagnosis of GCT did not clinically correlate with the patient's presentation. However initial pathology on core biopsy showing positive staining for S100 and CD68, classic for GCTs, guided our treatment of this patient. Our patient was managed surgically with excisional biopsy yielding two masses in the right breast and one mass in the left breast. Final pathology confirmed the initial diagnosis of GCTs of the breasts, and the patient has had an uncomplicated postoperative course. Although GCTs of the breast typically present as solitary and painless masses, multifocal presentation and pain are possible. It is also important to rule out malignancy with excisional biopsy of the masses and take into account the possibility of upstaging. Furthermore, it may be warranted to explore underlying genetic conditions such as LEOPARD syndrome if patient presents with additional findings.
\end{abstract}

Keywords: Granular cell tumors (GCTs); excisional biopsy; breast mass; case report

Received: 05 March 2021; Accepted: 02 April 2021; Published: 30 June 2021.

doi: 10.21037/asj-21-9

View this article at: http://dx.doi.org/10.21037/asj-21-9

\section{Introduction}

Granular cell tumors (GCT) are rare, benign tumors thought to originate from the nervous system. They can arise in any body site but predominantly are found in the digestive tract, oral cavity, and skin. They typically present as soft tissue neoplasms and were first reported by Abrikossoff and referred to as "granular cell myoblastoma" in the tongue and pharynx (1). They typically stain positive for S100 which is a marker for Schwann cells and GCTs are thought to arise from peripheral nerves. Though the majority of GCTs arise in the tongue (40\%), GCTs have been observed in the breast in 5-6\% of reported cases (2). They may be confused with invasive ductal or lobular carcinoma radiographically and typically present as a solitary, nontender nodule in the upper inner quadrant of the breast. The appearance of GCTs on mammography can be variable but the majority present as irregular high density non calcified spiculated masses. Sonographically, these present as irregular masses with angular margins and posterior shadowing. GCT of the breast manifests in the intralobular breast stroma and follows the distribution pathway of the supraclavicular nerve with a tendency for malignant variants to spread through lymphatic and hematogenous routes (3). Age of presentation in women ranges from 17 to 74 years, but most common presentation occurs in ages $30-50$ years, based on literature review (4).

Though this is a tumor typically presenting in women, 


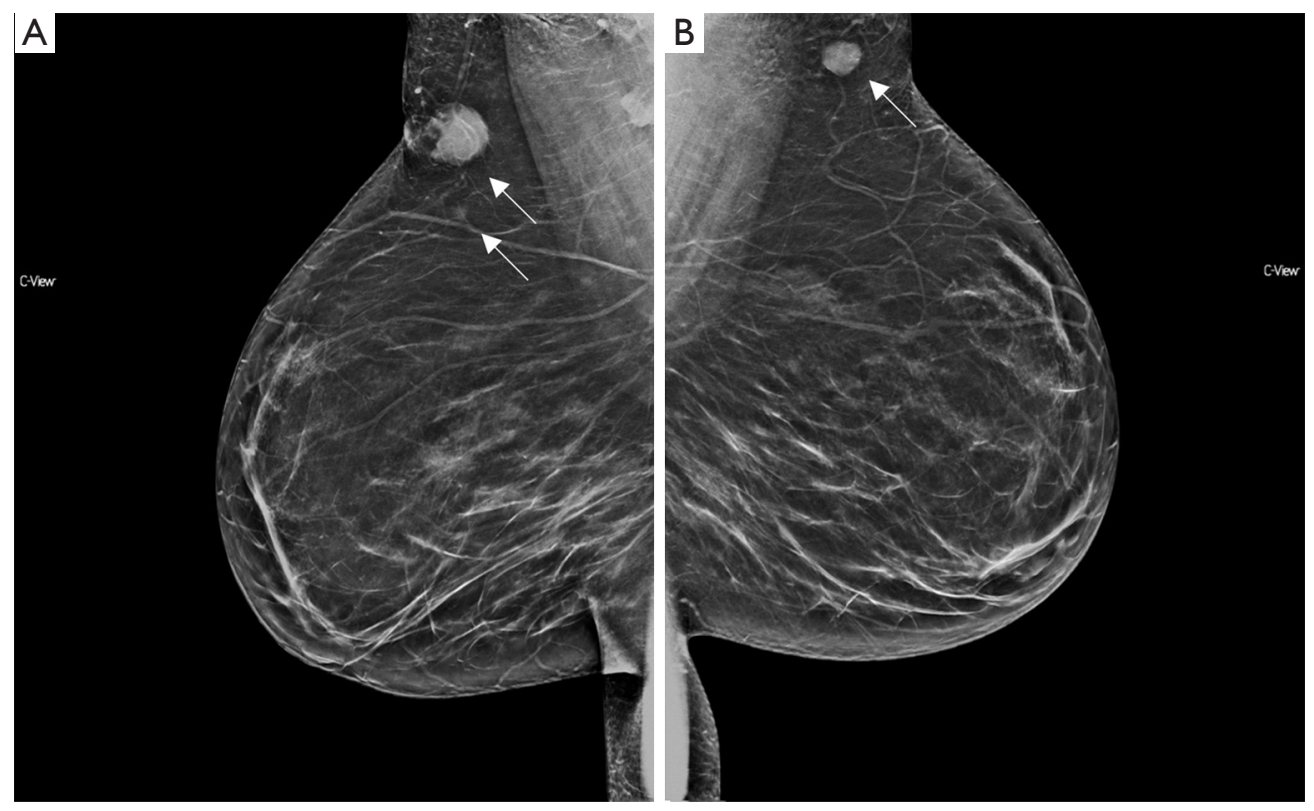

Figure 1 Bilateral medial-lateral oblique mammographic views. (A) Two irregular masses (arrows) with spiculated margins in the right breast at 1:00, $14 \mathrm{~cm}$ from the nipple; (B) round mass (arrow) with circumscribed margins in the left axillary tail.

it can occasionally occur in males. Literature review shows a handful of cases of GCTs in males aged 40-60 years who presented with a single palpable mass in one breast. In most of these cases, additional masses were also found in the abdominal wall, groin, or feet raising suspicion for possible conditions such as LEOPARD syndrome or PTEN mutations. Breast GCTs in men present similarly to women on mammography and sonography and positive S100 staining on pathology (5). Cases of multifocal GCTs involving just the breast are not well documented in the literature in males or females. We present the following case in accordance with the CARE reporting checklist (available at https://asj.amegroups.com/article/view/10.21037/asj-21$9 / \mathrm{rc})$.

\section{Case presentation}

We were asked to see a 39-year-old female with no significant medical or oncologic history who was referred to our clinic for a painful "knot" in her right breast with associated pain that increased during menstruation. This knot had been persistent for the past 4 years and she had not noticed any palpable masses elsewhere. Patient had not noticed any other breast changes including discoloration, nipple changes, contraction and also denied fever, chills or weight loss. Patient had a recent diagnostic mammographic evaluation which revealed two irregular masses with spiculated margins in the right breast at 11:00, $14 \mathrm{~cm}$ from the nipple, measuring 2.1 and $0.6 \mathrm{~cm}$ respectively, and a round mass with circumscribed margins in the axillary tail region of the left breast measuring $1.3 \mathrm{~cm}$ (Figure 1). Ultrasound-guided core biopsy of these 3 lesions (Figure 2) was consistent with GCTs of the breast with initial pathology showing classic large round to polygonal cells with abundant eosinophilic granular cytoplasm on hematoxylin and eosin stain, as well as tumor cells staining positive for S100 and CD68 (proteins classically expressed in GCTs). The patient was thoroughly counseled about the likely benign nature of GCTs but excision of all three lesions was recommended to rule out malignancy, relieve her symptoms and to prevent progression.

The patient was scheduled for surgery and underwent right breast excisional biopsy at two locations and left breast biopsy at one location. All three lesions were localized with Magseed localizers several days prior to surgery (Figure 3). After sterile preparation, the location of the dominant mass in the right breast was identified using palpation and a Sentimag device. Incision was made through the dermis and flaps were elevated with excision of tissue approximately $6 \mathrm{~cm} \times 4 \mathrm{~cm} \times 3 \mathrm{~cm}$ surrounding the mass. This excision extended down to the pectoralis muscle. After hemostasis was achieved, attention was focused on 

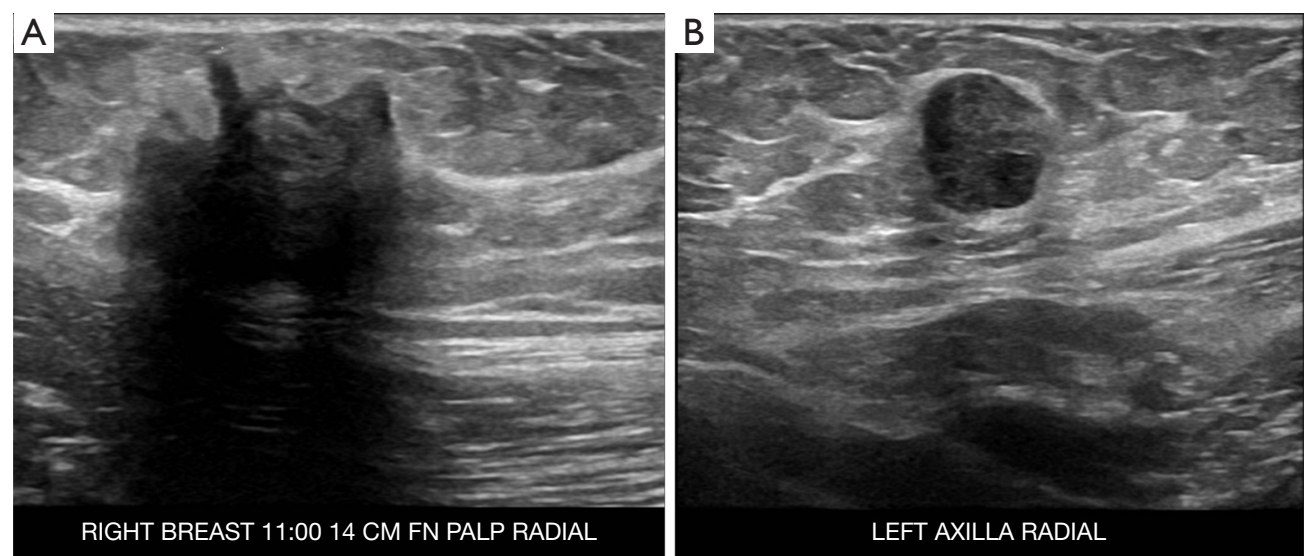

Figure 2 Diagnostic bilateral breast ultrasound. (A) Hypoechoic irregular mass with spiculated margins in the right breast mass at 11:00, $14 \mathrm{~cm}$ from the nipple measuring $2.1 \mathrm{~cm}$; (B) hypoechoic round mass with circumscribed margins in the axillary tail region of the left breast measuring $1.3 \mathrm{~cm}$.
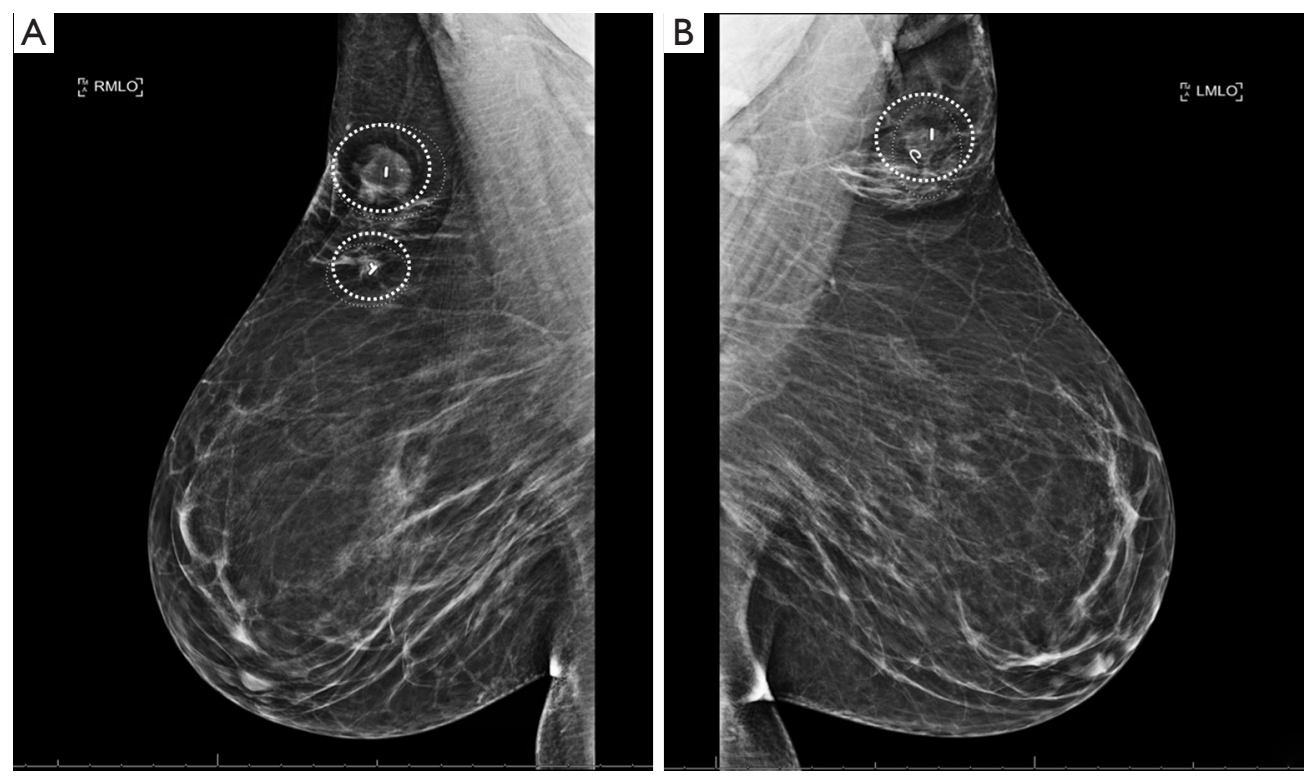

Figure 3 Bilateral medial-lateral oblique mammographic views following bilateral Magseed placement. (A) Two right breast masses with Magseed localizers (circles); (B) left breast mass with Magseed localizer (circle).

the smaller more medial mass in the right breast through the same incision, again using palpation and Sentimag for detection. Intra-operative specimen radiographs of the right breast tumors indicated well-centered masses, with the Magseeds in place and with normal surrounding tissue. The third and final mass in the left axillary tail region was then excised in a similar manner and sent to breast imaging, which confirmed removal of the mass with surrounding normal tissue and the Magseed in place. After closure, patient had a stable recovery course immediately postoperatively and was discharged the same day.

Gross examination of all three resection specimens showed gray-white well-circumscribed lesions ranging from $0.8 \mathrm{~cm}$ to largest $2.5 \mathrm{~cm}$. The Magseeds and the biopsy clips associated with the lesions were identified. Final pathology in the resected specimens from bilateral breasts showed a similar microscopic appearance of all three lesions. There were well-demarcated, poorly 


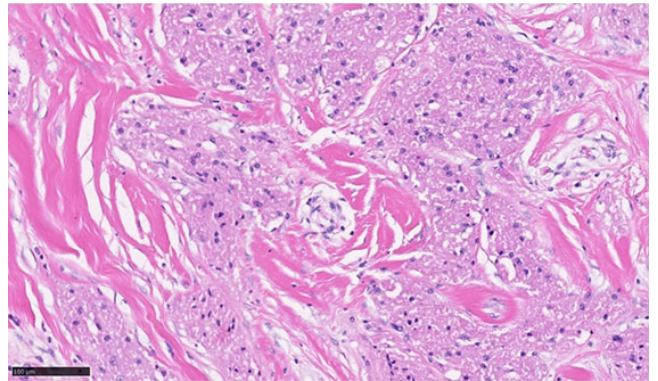

Figure 4 Representative hematoxylin and eosin staining image show nests of round to polygonal cells with abundant pale eosinophilic granular cytoplasm with inconspicuous borders and small bland nuclei considered as the hallmark of this tumor (magnification, 200×).

circumscribed, unencapsulated tumors composed of large round to polygonal cells in nests and sheets consisting of abundant eosinophilic granular cytoplasm (Figures 4,5), confirming the initial diagnosis of GCT. No histological features of invasive carcinoma were seen. The presence of associated biopsy site changes and negative surgical resection margins confirmed the complete excision of all three lesions.

Patient was seen for follow up visit on post-operative day 12 after the wide local excisions of the 3 breast lesions. Her only complaint at that time was persistent pain on the right side; on physical exam, her incisions were healing well, with no signs of seroma, hematoma, or infection. The patient was provided a more supportive bra, as her right sided pain was most likely secondary to her large breast placing tension on the incision site, and she was scheduled for a four month follow up visit in the clinic. Patient has had no emergent complaints four months post-operatively and had not been re-admitted for any complications or adverse reactions since initial surgery. Her overall postoperative course has been uncomplicated and patient will undergo repeat mammography in 1 year.

All procedures performed in this study were in accordance with the ethical standards of the institutional and/or national research committee(s) and with the Helsinki Declaration (as revised in 2013). Written informed consent was obtained from the patient for publication of this case report and accompanying images. A copy of the written consent is available for review by the editorial office of this journal.

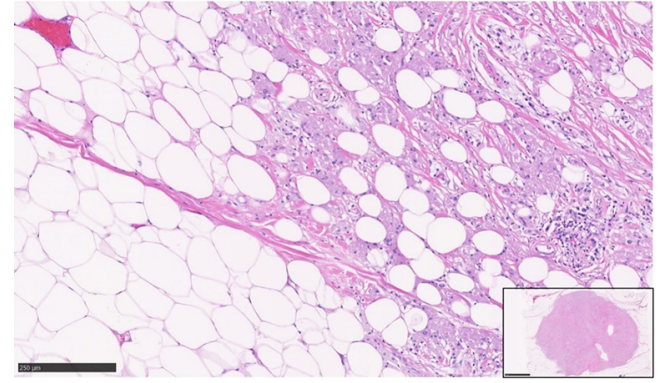

Figure 5 Representative hematoxylin and eosin staining image show infiltrative growth pattern of the tumor cells into the adjacent fibroadipose tissue (magnification, 100x) (inset, scanned image of the fairly well-demarcated tumor).

\section{Discussion}

This is an unusual presentation of breast GCTs based on location and multiplicity within both breasts. Based on literature review looking at case reports both from the United States and abroad, GCTs of the breast most commonly are solitary and present in the upper, inner quadrant of the breast (6). They can present as spiculated masses or well-circumscribed masses, but microcalcifications are not typical findings for GCTs (7). In addition, the majority of cases reported indicate that patients do not feel pain upon palpation of the mass. Our patient's presentation of painful masses in bilateral axillary regions makes her a unique case based on location, pain, and quantity, though radiographically is comparable to cases of breast GCT previously reported. This particular case allows us to understand the complexity of these tumors, and we now know that we cannot rule out such pathology based on atypical presentation alone.

Furthermore, radiologically and clinically, breast GCTs can mimic malignancies because of spiculated appearance and nipple and skin retraction on physical exam. In the case of breast GCTs alone, malignant GCTs account for only $2 \%$ of overall cases. However, literature shows reported cases of breast GCTs occurring synchronously with invasive ductal carcinoma (8). For this reason, it is crucial to keep in mind the possibility of this typically benign lesion being upstaged to malignancy. Moreover, because of the risk of local recurrence, they should be completely excised, preferably with negative 
margins. While GCTs of the breast are typically benign, they often mimic breast cancer on mammography and ultrasonography, which can lead to misdiagnosis. Incorrectly upstaging these masses can have a negative impact on patients including overtreatment and unnecessary surgical interventions. Fine-needle aspiration biopsy and frozen sections are inadequate for definitive diagnosis, but they are useful in differentiating between GCTs and breast carcinoma. Definitive diagnosis is made with immunohistochemical staining, and wide excision is sufficient for the treatment of GCTs (9). The existing literature shows that with excision, there is little longterm risk for recurrence even in instances where the mass was excised with positive or close $(<1 \mathrm{~mm})$ margins (10).

From a pathologic standpoint, grossly the tumor has a gray-white to tan appearance and is gritty to feel, giving an impression of carcinoma. Microscopic examination of all GCTs, irrespective of the site, is similar. The hallmarks are the poorly circumscribed, unencapsulated tumors composed of large round to polygonal cells in nests and sheets with abundant eosinophilic granular cytoplasm (Figure 4). Further, the ducts and lobules are typically entrapped amongst the infiltrative tumor cells (Figure 5). On electron microscopy, the granules are noted to be accumulated lysosomes. The most important differential diagnosis to exclude would be an invasive apocrine carcinoma, where cytokeratin stain will be positive, unlike GCTs. S100 is the most important immunohistochemical stain to confirm the GCT diagnosis, along with negative cytokeratin. The cytoplasmic granules can also be highlighted by a PAS (diastase resistant) histochemical stain. Most GCTs are benign, with extremely rare malignant GCTs reported in $0.5-2.0 \%$ of all GCTs (11). Although the criteria to determine malignancy are not clear, the FanburgSmith criteria comprising spindling cells, necrosis, large and vesicular nuclei, increased mitotic activity, high nuclear to cytoplasmic ratio and nuclear pleomorphism (at least 3 features required) are used (12). Recently, aberrant TFE3 expression was reported in GCTs of the kidney representing a degenerative change involving the cytoplasmic accumulation of lysosomes (13).

Furthermore, it is also important to keep in mind the possible germline genetic component of GCTs. Although they typically arise as benign solitary lesions, multiple GCTs can arise in up to $30 \%$ of cases. LEOPARD syndrome, a rare autosomal dominant syndrome, is characterized by multiple GCTs, ocular hypertelorism, obstructive cardiomyopathy, pulmonary stenosis, deafness, and multiple lentigines. LEOPARD syndrome is caused by a mutation in the PTPN11 gene which leads to dysregulation of the MAP kinase pathway, promoting increased cellular dedifferentiation and proliferation (14). Though our patient did not have any of these associated findings, genetic testing for this syndrome may be warranted for patients who clinically present with these additional features.

In conclusion, this case demonstrates an atypical presentation of breast GCTs in three separate locations within bilateral breasts. Surgical excision is the preferred approach to relieve symptoms, rule out malignancy and prevent progression. The possibility of upstaging to malignancy should always be considered when dealing with any breast mass, and genetic workup may be warranted based on the severity and location of GCTs. GCTs, though usually benign, may present with atypical findings such as pain or multifocality. Due to the rarity of this diagnosis, it may be easily overlooked, but this diagnosis should be considered when confronting multifocal breast lesions or additional syndromic findings.

\section{Acknowledgments}

Funding: None.

\section{Footnote}

Reporting Checklist: The authors have completed the CARE reporting checklist. Available at https://asj.amegroups.com/ article/view/10.21037/asj-21-9/rc

Conflicts of Interest: All authors have completed the ICMJE uniform disclosure form (available at https://asj.amegroups. com/article/view/10.21037/asj-21-9/coif). The authors have no conflicts of interest to declare.

Ethical Statement: The authors are accountable for all aspects of the work in ensuring that questions related to the accuracy or integrity of any part of the work are appropriately investigated and resolved. All procedures performed in this study were in accordance with the ethical standards of the institutional and/or national research committee(s) and with the Helsinki Declaration (as revised in 2013). Written informed consent was obtained from the patient for publication of this case report and accompanying images. A copy of the written consent is available for review 
by the editorial office of this journal.

Open Access Statement: This is an Open Access article distributed in accordance with the Creative Commons Attribution-NonCommercial-NoDerivs 4.0 International License (CC BY-NC-ND 4.0), which permits the noncommercial replication and distribution of the article with the strict proviso that no changes or edits are made and the original work is properly cited (including links to both the formal publication through the relevant DOI and the license). See: https://creativecommons.org/licenses/by-nc-nd/4.0/.

\section{References}

1. Akkaya H, Toru HS, Ayva ES, et al. Metachronous Occurrence of Granular Cell Tumor in Breast Skin and Scalp: Diagnostic Challenging Differentiating Benign from Malignant and a Literature Review. Case Rep Pathol 2016;2016:8043183.

2. Rexeena B, Paul A, Nitish RA, et al. Granular Cell Tumor of Breast: a Case Report and Review of Literature. Indian J Surg Oncol 2015;6:446-8.

3. Zeng Q, Liu L, Wen Q, et al. Imaging features of granular cell tumor in the breast: Case report. Medicine (Baltimore) 2020;99:e23264.

4. De Simone N, Aggon A, Christy C. Granular cell tumor of the breast: clinical and pathologic characteristics of a rare case in a 14-year-old girl. J Clin Oncol 2011;29:e656-7.

5. Patel HB, Leibman AJ. Granular cell tumor in a male breast: mammographic, sonographic, and pathologic features. J Clin Ultrasound 2013;41:119-21.

6. Fujiwara K, Maeda I, Mimura H. Granular cell tumor of the breast mimicking malignancy: a case

doi: 10.21037/asj-21-9

Cite this article as: Chilukuri S, Pillappa R, Allison K, Bear HD. Granular cell tumors of the breast: an unusual case of multifocality—a case report. AME Surg J 2021;1:7. report with a literature review. Acta Radiol Open 2018;7:2058460118816537.

7. Al-Balas M, De Leo A, Serra M, et al. Granular cell tumour of the breast: A rare presentation of a breast mass in an elderly female with a subsequent breast cancer diagnosis. SAGE Open Med Case Rep 2019;7:2050313X19841154.

8. Akahane K, Kato K, Ogiso S, et al. Malignant granular cell tumor of the breast: case report and literature review. Breast Cancer 2015;22:317-23.

9. Pergel A, Yucel AF, Karaca AS, et al. A therapeutic and diagnostic dilemma: granular cell tumor of the breast. Case Rep Med 2011;2011:972168.

10. Papalas JA, Wylie JD, Dash RC. Recurrence risk and margin status in granular cell tumors of the breast: a clinicopathologic study of 13 patients. Arch Pathol Lab Med 2011;135:890-5.

11. Elkousy H, Harrelson J, Dodd L, et al. Granular cell tumors of the extremities. Clin Orthop Relat Res 2000;(380):191-8.

12. Fanburg-Smith JC, Meis-Kindblom JM, Fante R, et al. Malignant granular cell tumor of soft tissue: diagnostic criteria and clinicopathologic correlation. Am J Surg Pathol 1998;22:779-94. Erratum in: Am J Surg Pathol 1999 Jan;23(1):136.

13. Liu Y, Zheng Q, Wang C, et al. Granular cell tumors overexpress TFE3 without gene rearrangement: Evaluation of immunohistochemistry and break-apart FISH in 45 cases. Oncol Lett 2019;18:6355-60.

14. Schrader KA, Nelson TN, De Luca A, et al. Multiple granular cell tumors are an associated feature of LEOPARD syndrome caused by mutation in PTPN11. Clin Genet 2009;75:185-9. 